\title{
Die Revision des
}

\section{ärztlichen Leistungstarifs}

\section{Klaus Völlm}

Dr. med., Facharzt für Kardiologie und Allgemeine Innere Medizin, Mitglied FMH

Schon lange dauert dieses Trauerspiel und droht in die Ausweglosigkeit bei fortschreitender Sonderteuerung im Gesundheitswesen zu führen. Die Meinungsgrenzen verlaufen quer durch die Gemeinschaft der niedergelassenen Ärzte und Ärztinnen (NGA): Spezialisten/Allgemeinpraktiker, Operierende/Nichtoperierende, Stadt-/Landärzte; ja sogar Standesorganisationen scheinen auf ihren Interessen beharren zu wollen. Neben den Fakten der demographischen Alterszunahme und der allgemeinen Teuerung lassen sich weitere Ursachen des Problems anführen:

\section{A)}

Bei der Einführung des TARMED wurde im Glauben, damit Missbrauch und Mengenausweitung verhindern zu können, die ärztliche Gesamtleistung in immer mehr einzelne Tarifpositionen aufgeteilt.

B)

Im folgenden Schema sollen die NGA unter einer statistischen Verteilungskurve in drei Gruppen eingeteilt werden.

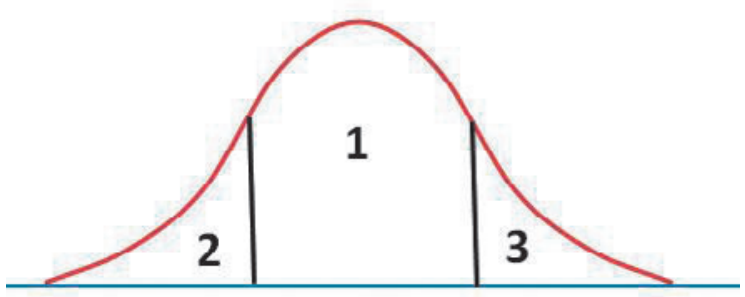

Einteilung der Gemeinschaft der niedergelassenen Ärzte und Ärztinnen (NGA) in drei Gruppen unter einer statistischen Verteilungskurve.

\section{Praxiseinkommen}

Gruppe 1 bilden verantwortungsvolle, korrekt abrechnende, «anständige» Ärzte und Ärztinnen. Ist es falsch, hier die Überzahl der NGA zu verorten, deren Wirken und Motivation Pflege verdient? In Gruppe 2 finden sich ökonomisch weniger Begabte und durch einen realen Einkommensrückgang Gefährdete. - In Gruppe 3 finden sich u.a. die "Numismatiker», eine Kategorie, die sich bis in eine Randgruppe unethisch bis unge- setzlich Praktizierender erstrecken kann. Bei der bisherigen Tarifregelung wurde unverständlicherweise die Gruppe 3 als repräsentativ und die gegen sie gemünzten Massnahmen als geeignet angesehen. Durch das Einfrieren, z.T. sogar die Herabsetzung des Tarifpunktwertes trotz allgemeiner Teuerung wird Gruppe 1 belastet (und Gr. 2 gefährdet). Dies stimuliert die Aktivitätsverlagerung auf lukrativere Positionen und eine Mengenausweitung. Wir wissen, dass dies manchenorts geschehen, Gruppen 1 und 2 «verdorben" und damit das angeblich kostenlimitierende TARMED-System kompensiert worden ist. Die Ärzteschaft entkräftet damit selbst ihren Vorwurf eines fehlenden Teuerungsausgleichs.

\section{C)}

(Das Folgende dürfte nicht überall gerne gehört werden.) Der NGA geniesst in der Gesellschaft immer noch und nicht nur ein hohes Ansehen. Mit der Praxisbewilligung, dem Kassenobligatorium und dem Versicherungs-Vertragszwang erhält er auch staatlich-rechtlich garantierte Privilegien - ein überdurchschnittliches Einkommen und Arbeitssicherheit -, die einer Arztpraxis den Charakter eines geschützten Arbeitsplatzes geben. Staatsangestellte, bis hinauf zum Bundesrat, werden nach einem einsehbaren Tarifsystem entlohnt. Einzelne Ärzte sollen es bis zum Mehrfachen eines Bundesratssalärs schaffen - und können nicht abgewählt werden. Die Einkommensverhältnisse der NGA sind weitgehend intransparent und werden - unter der Illusion eines immer noch freien Berufsstandes -streng gehütet.

Wer seit Jahren von staatlichen Privilegien profitiert hat, kann konsequenterweise nicht ein öffentliches Interesse an der gerechten Vergütung seiner Leistungen ablehnen. Der Forderung einer vermehrten Transparenz des Ärzteeinkommens wird wohl mit dem Vorwurf einer Verstaatlichung eines Berufsstandes begegnet. Tatsächlich sind wir schon halbwegs dort.

D)

Zu den Ursachen gehört auch die mit dem TARMED erfolgte Abschaffung der Privatmedizin in der ambulanten Praxis. Das Nebeneinander von obligatorisch und 
privat versicherten Patienten gab dem Arzt die Möglichkeit, in seiner Praxis, wie in einer kleinen sozialen Einheit, bei qualitativ gleichwertiger ärztlicher Behandlung einen Ausgleich zwischen dem Einkommen aus den beiden Gruppen zu schaffen. Mit dem Verbot der Privathonorierung ist in manchen Praxen schlagartig ein ganz wesentlicher Einkommensteil weggefallen: ein weiteres Motiv zur Ausschöpfung des TARMED. Dies wäre Aussperrung eines privaten Geldzuflusses zu Ungunsten des Staatshaushaltes.

Beim Verbot der Privathonorierung und den überschiessenden Massnahmen spielte zweifellos die Verdächtigung der Ärzte unfairer Selbsthonorierung und Diskriminierung der obligatorisch Versicherten mit: ein politisches Beispiel eingreifender Reglementierung aufgrund "qualitativer», zwar vorhandener, aber hochgespielter Argumente, ohne quantitative Berücksichtigung von Wirkungen und Nebenwirkungen.

\section{Wer kritisiert, sollte Gegenvorschläge machen}

Meine Anregungen zielen zunächst auf eine Trennung der Tarifgestaltung für persönliche ärztliche und für technische Leistungen, ohne auf eine Motivation zum Angebot letzterer ganz zu verzichten.

1. Jeder NGA arbeitet durchgehend zu einem Zeittarif, womit sein persönlicher Einsatz erfasst wird. Ärzten mit längerer qualifizierter Ausbildung (A/BKliniken, FMH-Titel, dokumentierte Fähigkeit und Erfahrung in der Anwendung spezieller Techniken) wird die längere Weiterbildung honoriert. NGA mit klassifizierter Weiterbildung würden zum gleichen Tarif arbeiten, ob sie an einer besonderen Apparatur oder in der Patientenberatung tätig sind, denn auch in die Beratung fliesst die Spezialerfahrung ein. Invasive Leistungen, vorwiegend im therapeutischen und chirurgischen Bereich, bei denen Fähigkeit und Erfahrung ein speditives und atraumatischeres Vorgehen erlauben, verdienen eine besondere Dignität. Sie erlauben effizienteres Zeitmanagement, auf der Nutzenseite für den Patienten steht eine kürzere und schmerzärmere Rekonvaleszenz. Der Grundwert jeder Tarifklasse wird wie bisher abgestuft nach den Arbeitsumständen: RoutinePraxiszeit, Wochenende und Nachteinsatz, Notfall. Die Schaffung eines als gerecht und sinnvoll empfundenen und akzeptanzfähigen Tarifpunktsystems wird die anspruchsvollste Aufgabe der Revision sein.

2. Alle technisch-apparativen Leistungen sind auf der Basis der Selbstkosten abzurechnen, mit dem Zuschlag einer gegenüber der bisherigen Usanz deutlich reduzierten Gewinnmarge. Die Vergütung des einzelnen Apparate-Einsatzes wäre aufgrund diverser klarer Jahresabrechnungen zu ermitteln, unter
Berücksichtigung von Amortisation, Unterhalt, Platzbedarf der Anlage sowie Personalkosten.

3. Alle Bemühungen um eine adäquate Honorierung medizinischer Leistungen laufen ins Ungewisse, wenn die Umsetzung des Reglements beim Leistungserbringer nicht einsehbar ist. Es sollte möglich werden, dass eine Instanz, die das Arztgeheimnis garantiert, Einblick in die Abrechnungsweise eines Praxisinhabers erhält. NGA wären zu einer separaten Buchführung aller Zahlungseingänge von allgemein versicherten Patienten zu verpflichten. Bei Verdacht auf unkorrekte Verrechnung, aber auch als randomisierte Stichproben, müssten diese Zahlungseingänge und relevante Einzelrechnungen vorgelegt werden.

\section{Kommentare}

ad 1. Wenn die Honorierung persönlicher Leistungen des Arztes vermehrt an ihren Zeitaufwand gekoppelt wird, liesse sich eine Mengenausweitung in dem Bereich, der am meisten zum Arzteinkommen beitragen sollte, an einem unwahrscheinlichen Gesamtzeitaufwand erkennen.

ad 2. Eine moderate Gewinnmarge im rein technischen Bereich hilft, das Risiko teurerer Anschaffungen zu reduzieren, und bildet einen Anreiz, fortschrittliche Behandlungen anzubieten.

ad 3. Nur Leistungsvergütungen obligatorisch Versicherter lassen sich durch ein Regulativ einfach kontrollieren. Zahlungen aus privaten Zusatzversicherungen oder von selbstzahlenden Patienten beruhen auf einem freiwilligen Vertragsverhältnis. Gesetzlich sind Privatrechnungen nur für stationäre Behandlungen auf Privatabteilungen oder in Privatspitälern zulässig und somit nur einer Minderheit der NGA möglich.

Forderungen nach Offenlegung im Verrechnungsbereich stossen in der Ärzteschaft stets auf grossen Widerstand. Ihre Akzeptanz würde ein grosses Umdenken erfordern, sowie die Einsicht, dass die Gesundheitspflege tatsächlich eine staatliche Angelegenheit geworden ist.

Kein Tarifsystem kann einzelne Ungerechtigkeiten ausschliessen. Die bisherige Gesundheitspolitik hat aber im ambulanten und stationären Bereich ihr Ziel einer effektiven Kostenkontrolle nicht erreicht. Eine Tarifrevision nach neuen Grundsätzen ist in hohem Masse von der Kooperation und Kompromissbereitschaft der NGA abhängig. Die bisherige Linie führt zu Blockierung und Chaos unter den zersplitterten Interessengruppen und letztlich zum etatistischen Diktat. Zuletzt sei nochmals darauf hingewiesen, dass in er heutigen Zeit bald die Forderung nach vermehrter Transparenz der ärztlichen Einkommensverhältnisse zu erwarten und berechtigt ist. 